ISSN 1855-3966 (printed edn.), ISSN 1855-3974 (electronic edn.)

\author{
ARS MATHEMATICA CONTEMPORANEA 20 (2021) 151-170 \\ https://doi.org/10.26493/1855-3974.2201.b65 \\ (Also available at http://amc-journal.eu)
}

\title{
Geometry of the parallelism in polar spine spaces and their line reducts
}

\author{
Krzysztof Petelczyc (D), Krzysztof Prażmowski (D), Mariusz Żynel (D) \\ Faculty of Mathematics, University of Biatystok, \\ Ciołkowskiego 1 M, 15-245 Biatystok, Poland
}

Received 18 December 2019, accepted 22 November 2020, published online 20 October 2021

\begin{abstract}
The concept of the spine geometry over a polar Grassmann space belongs to a wide family of partial affine line spaces. It is known that the geometry of a spine space over a projective Grassmann space can be developed in terms of points, so called affine lines, and their parallelism (in this case the parallelism is not intrinsically definable as it is not Veblenian). This paper aims to prove an analogous result for the polar spine spaces. As a by-product we obtain several other results on primitive notions for the geometry of polar spine spaces.
\end{abstract}

Keywords: Grassmann space, projective space, polar space, spine space, coplanarity, pencil of lines. Math. Subj. Class. (2020): 51A15, 51A45

\section{Introduction}

Some properties of the polar spine spaces were already established in [8], where the class of such spaces was originally introduced. Its definition resembles the definition of a spine space defined within a (projective) Grassmann space (= the space $\mathbf{P}_{k}(\mathbb{V})$ of pencils of $k$-subspaces in a fixed vector space $\mathbb{V}$ ), cf. [12, 13]. In every case, a spine space is a fragment of a (projective) Grassmannian whose points are subspaces which intersect a fixed subspace $W$ in a fixed dimension $m$. In case of polar spine spaces we consider a twostep construction, in fact: we consider the subspaces of $\mathbb{V}$ that are totally isotropic (self conjugate, singular) under a fixed nondegenerate reflexive bilinear form $\xi$ on $\mathbb{V}$, and then we restrict this class to the subspaces which touch $W$ in dimension $m$.

It is a picture which is seen from the view of $\mathbb{V}$. Clearly, $W$ can be extended to a subspace $M$ of $\mathbb{V}$ with codimension 1 and then $M$ yields a hyperplane $\mathcal{M}$ of the polar space

E-mail addresses: kryzpet@math.uwb.edu.pl (Krzysztof Petelczyc), krzypraz@math.uwb.edu.pl (Krzysztof Prażmowski), mariusz@math.uwb.edu.pl (Mariusz Żynel) 
$\mathrm{Q}_{0}$ determined by $\xi$ in $\mathbb{V}$. In other words, the projective points on $W$ that are points of $\mathrm{Q}_{0}$ yield a subspace $\mathcal{W}$ of $Q_{0}$ extendable to a hyperplane. Recall that situation of this sort was already investigated in [10]. The isotropic $k$-subspaces of $\mathbb{V}$ are the $(k-1)$-dimensional linear subspaces of $\mathrm{Q}_{0}$, and first-step restriction yields the so called polar Grassmann space $\mathrm{Q}_{k-1}=\mathbf{P}_{k-1}(\mathrm{Q})$. The points of $\mathrm{Q}_{k-1}$ which touch $W$ in dimension $m$ are - from view of $\mathrm{Q}$ - the elements of $\mathrm{Q}_{k-1}$ which touch $\mathcal{W}$ in dimension $m-1$. So, a polar spine space is also the fragment of a polar Grassmannian which consists of subspaces which touch a fixed subspace extendable to a hyperplane in a fixed dimension. The analogy seems full.

In particular, when $W$ is a hyperplane of $\mathbb{V}$ i.e. $\mathcal{W}$ is a hyperplane of $Q_{0}$ then a $k$ subspace of $\mathrm{Q}$ either is contained in $\mathcal{W}$ or it touches it in dimension $k-1$. It is seen that in this case the only reasonable value of $m$ is $m=k-1$ and the obtained structure is the Grassmannian of subspaces of the affine polar space obtained from $Q_{0}$ by deleting $\mathcal{W}$ (cf. [3, 11]). So, the class of polar spine spaces contain Grassmannians of $k$-subspaces of arbitrary polar slit space: of a polar space with a subspace (extendable to a hyperplane) removed, see [10]. An interesting case appears, in particular, when we assume that $W$ is isotropic.

\section{Generalities}

This section is quoted after [8] with slight modifications.

\subsection{Point-line spaces and their fragments}

A point-line structure $\mathfrak{B}=\langle S, \mathcal{L}\rangle$, where the elements of $S$ are called points, the elements of $\mathcal{L}$ are called lines, and where $\mathcal{L} \subset 2^{S}$, is said to be a partial linear space, or a point-line space, if two distinct lines share at most one point and every line is of size (cardinality) at least 2 (cf. [2]).

A subspace of $\mathfrak{B}$ is any set $X \subseteq S$ with the property that every line which shares with $X$ two or more points is entirely contained in $X$. We say that a subspace $X$ of $\mathfrak{B}$ is strong if any two points in $X$ are collinear. If $S$ is strong, then $\mathfrak{B}$ is said to be a linear space.

Let us fix a nonempty subset $\mathcal{H} \subset S$ and consider the set

$$
\left.\mathcal{L}\right|_{\mathcal{H}}:=\{k \cap \mathcal{H}: k \in \mathcal{L} \text { and }|k \cap \mathcal{H}| \geq 2\} .
$$

The structure

$$
\mathfrak{M}:=\mathfrak{B}\left\lceil\mathcal{H}=\left\langle\mathcal{H},\left.\mathcal{L}\right|_{\mathcal{H}}\right\rangle\right.
$$

is a fragment of $\mathfrak{B}$ induced by $\mathcal{H}$ and itself it is a partial linear space. The incidence relation in $\mathfrak{M}$ is again $\in$, inherited from $\mathfrak{B}$, but limited to the new point set and line set. Following a standard convention we call the points of $\mathfrak{M}$ proper, and the points in $S \backslash \mathcal{H}$ improper. The set $S \backslash \mathcal{H}$ will be called the horizon of $\mathfrak{M}$. To every line $\left.L \in \mathcal{L}\right|_{\mathcal{H}}$ we can assign uniquely the line $\bar{L} \in \mathcal{L}$, the closure of $L$, such that $L \subseteq \bar{L}$. For a subspace $X \subseteq \mathcal{H}$ the closure of $X$ is the minimal subspace $\bar{X}$ of $\mathfrak{B}$ containing $X$. A line $\left.L \in \mathcal{L}\right|_{\mathcal{H}}$ is said to be a projective line if $L=\bar{L}$, and it is said to be an affine line if $|\bar{L} \backslash L|=1$. With every affine line $L$ one can correlate the point $L^{\infty} \in S \backslash \mathcal{H}$ by the condition $L^{\infty} \in \bar{L} \backslash L$. We write $\mathcal{A}$ for the class of affine lines. In what follows we consider sets $\mathcal{H}$ which satisfy the following

$$
|L \backslash \mathcal{H}| \leq 1 \text { or }|L \cap \mathcal{H}| \leq 1 \text { for all } L \in \mathcal{L} .
$$

Note that the above holds when $\mathcal{H}$ or $S \backslash \mathcal{H}$ is a subspace of $\mathfrak{B}$, but the above does not force $\mathcal{H}$ or $S \backslash \mathcal{H}$ to be a subspace of $\mathfrak{B}$. In any case, under this assumption every line 
is either projective or affine. In case $\left.\mathcal{L}\right|_{\mathcal{H}}$ contains projective or affine lines only, then $\mathfrak{M}$ is a semiaffine geometry (for details on terminology and axiom systems see [18]). In this approach an affine space is a particular case of a semiaffine space. For affine lines $L_{1},\left.L_{2} \in \mathcal{L}\right|_{\mathcal{H}}$ we can define a parallelism in a natural way:

$$
L_{1}, L_{2} \text { are parallel }\left(L_{1} \| L_{2}\right) \text { iff } \overline{L_{1}} \cap \overline{L_{2}} \cap(S \backslash \mathcal{H}) \neq \emptyset .
$$

In what follows we assume that the notion of 'a plane' (= 2-dimensional strong subspace) is meaningful in $\mathfrak{B}$ : e.g. $\mathfrak{B}$ is an exchange space, or a dimension function is defined on its strong subspaces. In the article in most parts we consider $\mathfrak{B}$ such that its planes are, up to an isomorphism, projective planes. We say that $E$ is a plane in $\mathfrak{M}$ if $\bar{E}$ is a plane in $\mathfrak{B}$. Observe that there are two types of planes in $\mathfrak{M}$ : projective and semiaffine. A semiaffine plane $E$ arises from $\bar{E}$ by removing a point or a line. In result we get a punctured plane or an affine plane respectively. For lines $L_{1},\left.L_{2} \in \mathcal{L}\right|_{\mathcal{H}}$ we say that they are coplanar and write

$$
L_{1} \pi L_{2} \text { iff there is a plane } E \text { such that } L_{1}, L_{2} \subset E \text {. }
$$

Let $E$ be a plane in $\mathfrak{M}$ and $U \in \bar{E}$. The set

$$
\mathbf{p}(U, E):=\left\{\left.L \in \mathcal{L}\right|_{\mathcal{H}}: U \in \bar{L} \subseteq \bar{E}\right\}
$$

will be called a pencil of lines if $U$ is a proper point, or a parallel pencil otherwise. The point $U$ is said to be the vertex and the plane $E$ is said to be the base plane of that pencil. We write

$$
L_{1} \rho L_{2} \quad \text { iff } \quad \text { there is a pencil } p \text { such that } L_{1}, L_{2} \in p \text {. }
$$

\subsection{Cliques}

Let $\varrho$ be a binary symmetric relation defined on a set $X$. A subset of $X$ is said to be a $\varrho$-clique iff every two elements of this set are $\varrho$-related.

For any $x_{1}, x_{2}, \ldots, x_{s}$ in $X$ we introduce

$$
\begin{array}{r}
\boldsymbol{\Delta}_{\varrho}^{s}\left(x_{1}, x_{2}, \ldots, x_{s}\right) \text { iff } \neq\left(x_{1}, x_{2}, \ldots, x_{s}\right) \text { and } x_{i} \varrho x_{j} \text { for all } i, j=1, \ldots, s \\
\text { and for all } y_{1}, y_{2} \in X \text { if } y_{1}, y_{2} \varrho x_{1}, x_{2}, \ldots, x_{s} \text { then } y_{1} \varrho y_{2},
\end{array}
$$

cf. analogous definition of $\Delta_{s}^{\varrho}$ in [9]. For short we will frequently write $\Delta_{\varrho}$ instead of $\Delta_{\varrho}^{s}$. Next, we define

$$
\left[x_{1}, x_{2}, \ldots, x_{s}\right]_{\varrho}:=\left\{y \in X: y \varrho x_{1}, x_{2}, \ldots x_{s}\right\} .
$$

It is evident that if $\boldsymbol{\Delta}_{\varrho}\left(x_{1}, \ldots, x_{s}\right)$ holds (and $\varrho$ is reflexive) then $\left[x_{1}, \ldots, x_{s}\right]_{\varrho}$ is the (unique) maximal $\varrho$-clique which contains $\left\{x_{1}, \ldots, x_{s}\right\}$. Finally, for an arbitrary integer $s \geq 3$ we put

$$
\mathcal{K}_{\varrho}^{s}=\left\{\left[x_{1}, x_{2}, \ldots, x_{s}\right]_{\varrho}: x_{1}, x_{2}, \ldots, x_{s} \in \mathcal{X} \text { and } \boldsymbol{\Delta}_{\varrho}\left(x_{1}, x_{2}, \ldots, x_{s}\right)\right\} .
$$

Then we write

$$
\mathcal{K}_{\varrho}:=\bigcup_{s=3}^{\infty} \mathcal{K}_{\varrho}^{s} .
$$

In most of the interesting situations there is an integer $s_{\max }$ such that $\mathcal{K}_{\varrho}=\bigcup_{s=3}^{s_{\max }} \mathcal{K}_{\varrho}^{s}=$ $\mathcal{K}^{*}(\varrho)$, where

$$
\mathcal{K}^{*}(\varrho) \text { is the set of maximal } \varrho \text {-cliques. }
$$




\subsection{Grassmann spaces and spine spaces}

We start with some constructions of a general character. Let $X$ be a nonempty set and let $\mathcal{P}$ be a family of subsets of $X$. Assume that there is a dimension function $\operatorname{dim}: \mathcal{P} \rightarrow$ $\{0, \ldots, n\}$ such that $\mathfrak{B}=\langle\mathcal{P}, \subset, \operatorname{dim}\rangle$ is an incidence geometry, cf. e.g. [1]. Write $\mathcal{P}_{k}$ for the set of all $U \in \mathcal{P}$ with $\operatorname{dim}(U)=k$.

Given $H \in \mathcal{P}_{k-1}$ and $B \in \mathcal{P}_{k+1}$ with $H \subset B$, a $k$-pencil over $\mathfrak{B}$ is a set of the form

$$
\mathbf{p}(H, B)=\left\{U \in \mathcal{P}_{k}: H \subset U \subset B\right\} .
$$

The idea behind this concept is the same as in (1.3), though this definition is more general. The family of all such $k$-pencils over $\mathfrak{B}$ will be denoted by $\mathcal{P}_{k}$. Then, the structure

$$
\mathbf{P}_{k}(\mathfrak{B})=\left\langle\mathcal{P}_{k}, \mathcal{P}_{k}\right\rangle
$$

will be called a Grassmann space over $\mathfrak{B}$ (cf. [5, Section 2.1.3]). It is a partial linear space for $0<k<n$.

Let us fix $W \in \mathcal{P}$ and an integer $m$. We will write

$$
\mathcal{F}_{k, m}(\mathfrak{B}, W):=\left\{U \in \mathcal{P}_{k}: \operatorname{dim}(U \cap W)=m\right\} .
$$

The fragment

$$
\mathbf{A}_{k, m}(\mathfrak{B}, W):=\mathbf{P}_{k}(\mathfrak{B}) \uparrow \mathcal{F}_{k, m}(\mathfrak{B}, W)
$$

will be called a spine space over $\mathfrak{B}$ determined by $W$. It will be convenient to have an additional symbol for the line set of a spine space, which is

$$
\mathcal{G}_{k, m}(\mathfrak{B}, W):=\left.\mathcal{P}_{k}\right|_{\mathcal{F}_{k, m}(\mathfrak{B}, W)}
$$

What follows are more specific examples of the above constructions that we actually investigate in our paper. Let $\mathbb{V}$ be a vector space and let $\operatorname{Sub}(\mathbb{V})$ be the set of all vector subspaces of $\mathbb{V}$. Then $\mathbf{P}_{k}(\mathbb{V})$ is a partial linear space called a projective Grassmann space. In particular $\mathbf{P}_{1}(\mathbb{V})$ is the projective space over $\mathbb{V}$. It is well known that $\mathbf{P}_{k}(\mathbb{V}) \cong \mathbf{P}_{k-1}\left(\mathbf{P}_{1}(\mathbb{V})\right)$.

Let $W \in \operatorname{Sub}(\mathbb{V})$. The spine space $\mathbf{A}_{k, m}(\mathbb{V}, W)$ was introduced in [12] and developed in $[13,14,15,16]$. Note that $\mathbf{A}_{k, m}(\mathbb{V}, W) \cong \mathbf{A}_{k-1, m-1}\left(\mathbf{P}_{1}(\mathbb{V}), \operatorname{Sub}_{1}(W)\right)$. The concept of a spine space makes a little sense without the assumption that

$$
0, k-n+w \leq m \leq k, w
$$

where $w=\operatorname{dim}(W)$. It is a partial linear space when (1.8) is satisfied.

For possibly maximal values of $m$ we get $\mathbf{A}_{k, k}(\mathbb{V}, W)=\mathbf{P}_{k}(W)$, where the points are basically vector subspaces of $W$, and $\mathbf{A}_{k, w}(\mathbb{V}, W) \cong \mathbf{P}_{k-w}(\mathbb{V} / W)$, where the points are those vector subspaces of $\mathbb{V}$ which contain $W$. Therefore, we assume that

$$
m<k, w
$$

Now, let $\xi$ be a nondegenerate reflexive bilinear form of index $r$ on $\mathbb{V}$. For $U, W \in$ $\operatorname{Sub}(\mathbb{V})$ we write $U \perp W$ iff $\xi(U, W)=0$, meaning that $\xi(u, w)=0$ for all $u \in U$, $w \in W$. Then the set of all totally isotropic subspaces of $\mathbb{V}$ w.r.t. $\xi$ is

$$
\mathrm{Q}:=\{U \in \operatorname{Sub}(\mathbb{V}): U \perp U\},
$$


and $\mathrm{Q}_{k}:=\mathrm{Q} \cap \operatorname{Sub}_{k}(\mathbb{V})$. The set $\mathrm{Q}_{k}$ is nonempty iff

$$
k \leq r
$$

Provided that $2 \leq r$ the structure $\mathfrak{Q}=\mathbf{P}_{1}(\mathrm{Q})$ is a classical polar space embeddable into the projective space $\mathbf{P}_{1}(\mathbb{V})$. It is clear that $\mathfrak{Q} \cong\left\langle Q_{1}, Q_{2}, C\right\rangle$ and usually polar space is defined that way.

A polar Grassmann space is the structure $\mathbf{P}_{k}(\mathrm{Q})$. It is a partial linear space whenever

$$
k<r \text {. }
$$

Note that $\mathbf{P}_{k}(\mathrm{Q}) \cong \mathbf{P}_{k-1}\left(\mathbf{P}_{1}(\mathrm{Q})\right)$.

Finally,

$$
\mathfrak{M}:=\mathbf{A}_{k, m}(\mathrm{Q}, W),
$$

a polar spine space, the main subject of our paper, arises. Note that we have $\mathfrak{M} \cong$ $\mathbf{A}_{k-1, m-1}\left(\mathbf{P}_{1}(\mathrm{Q}), \operatorname{Sub}(W) \cap \mathrm{Q}_{1}\right)$.

Let $r_{W}=\operatorname{ind}\left(\xi\lceil W)\right.$ be the index of the form $\xi$ restricted to $W$. If $r_{W}<m$, then there is no totally isotropic subspace of $\mathbb{V}$, which meets $W$ in some $m$-dimensional subspace. Every $U \in \mathrm{Q}$ can be extended to an $Y \in \mathrm{Q}_{r}$. Assume that $\operatorname{dim}(Y \cap W)>r-k+m$ for all $Y \in \mathrm{Q}_{r}$. This means that all totally isotropic subspaces of $\mathbb{V}$, which meet $W$ in some $m$-dimensional subspace, are at most $(k-1)$-dimensional. On the other hand, this assumption implies $r_{W}>r-k+m$. Thus

$$
m \leq r_{W} \leq r-k+m
$$

is a sufficient condition for $\mathcal{F}_{k, m}(\mathrm{Q}, W) \neq \emptyset$.

Warning. The condition (1.12) is - in the context above - only sufficient. As we shall see there are sets $W$ such that $r-k+m<r_{W}$ but $\mathcal{F}_{k, m}(\mathrm{Q}, W) \neq \emptyset$. Clearly, the condition $m \leq r_{W}$ is necessary.

Under (1.12) no point of $\mathfrak{M}$ is isolated and $\mathfrak{M}$ is a partial linear space. Now, let us have a look at the structure of strong subspaces of polar spine spaces. Following [13] they are called: $\alpha$-stars, $\omega$-stars, $\alpha$-tops and $\omega$-tops. For details see Table 2 . Actually, this is an 'adaptation' of the classification of strong subspaces of $\mathbf{A}_{k, m}(\mathbb{V}, W)$ (consult [13]) to the case when we restrict $\mathbf{P}_{k}(\mathbb{V})$ to $\mathbf{P}_{k}(\mathrm{Q})$. With a slight abuse of language all sets of the type $\mathcal{T}^{\alpha}$ and $\mathcal{T}^{\omega}$ we call tops, and sets of the form $\mathcal{S}^{\alpha}$ and $\mathcal{S}^{\omega}$ stars. But note that due to some specific values of $r, k, m$ and $\operatorname{dim}(Y \cap W)$ with $Y \in \mathrm{Q}_{r}$ families of some of these types may be empty. Moreover, stars and tops consist of strong subspaces of $\mathfrak{M}$, but stars or tops of some kind may be not maximal among strong. In general, $\mathcal{S}^{\omega}$ and $\mathcal{T}^{\alpha}$ consist of projective spaces, while the other consist of proper slit spaces (cf. [4, 18]), but if $\mathcal{F}_{k-1, m}(\mathrm{Q}, W) \ni H \subset Y \in \mathcal{F}_{r, m}(\mathrm{Q}, W)$ then $[H, Y]_{k} \cap \mathcal{F}_{k, m}(\mathrm{Q}, W)=[H, Y]_{k} \in \mathcal{S}^{\alpha}$ is a projective space as well.

Generally, $H \in \mathcal{P}_{k-1}$ determines a star and $B \in \mathcal{P}_{k+1}$ determines a top as follows

$$
\mathrm{S}(H)=\left\{U \in \mathcal{P}_{k}: H \subset U\right\}, \quad \mathrm{T}(B)=\left\{U \in \mathcal{P}_{k}: U \subset B\right\} .
$$

Here, we occasionally make use of this convention in the context of polar spine spaces, where $\mathcal{P}_{k}=\mathcal{F}_{k, m}(\mathrm{Q}, W)$. 


\section{Lines classification and existence problems}

In analogy to $[13,17]$ the lines of $\mathfrak{M}$ can be of three sorts: affine (in $\mathcal{A}$ ), $\alpha$-projective (in $\mathcal{L}^{\alpha}$ ), and $\omega$-projective (in $\mathcal{L}^{\omega}$ ). To be more concrete, comp. Table 1 , these are pencils $L=\mathbf{p}(H, B) \cap \mathcal{F}_{k, m}(\mathrm{Q}, W)$ such that (we consider parameters $k, m, \mathrm{Q}, W$ as fixed)

$\mathcal{A}: \quad H \in \mathcal{F}_{k-1, m}(\mathrm{Q}, W), B \in \mathcal{F}_{k+1, m+1}(\mathrm{Q}, W)$; in this case $L^{\infty}=H+(W \cap B)=$ $(H+W) \cap B$. Note that $L^{\infty} \subset B \in \mathrm{Q}$ and therefore $L^{\infty} \in \mathcal{F}_{k, m+1}(\mathrm{Q}, W)$. In other words, $L^{\infty}$ is a point of $\mathbf{A}_{k, m+1}(\mathrm{Q}, W)$.

$\mathcal{L}^{\alpha}: \quad H \in \mathcal{F}_{k-1, m}(\mathrm{Q}, W), B \in \mathcal{F}_{k+1, m}(\mathrm{Q}, W)$.

$\mathcal{L}^{\omega}: \quad H \in \mathcal{F}_{k-1, m-1}(\mathrm{Q}, W), B \in \mathcal{F}_{k+1, m+1}(\mathrm{Q}, W)$

(cf. Table 1).

Note that if $r_{W}<m+1$ (in view of the global assumption $r_{W} \geq m$ this means $r_{W}=m$ ) then $\mathcal{A} \cup \mathcal{L}^{\omega}=\emptyset$. Looking at [8, Lemma 1.6] we see that in this case $\mathfrak{M}$ is disconnected as well or $m=w$. In the latter case also $\mathcal{A} \cup \mathcal{L}^{\omega}=\emptyset$. Besides, this also contradicts (1.9). Consequently, for $r_{W}<m+1$ the horizon $\mathbf{A}_{k, m+1}(\mathrm{Q}, W)$ of $\mathfrak{M}$ looses its sense.

The problem whether one of the three above classes of lines is nonempty reduces, in fact, to the problem whether the corresponding class of 'possible tops' of these lines is nonempty. More precisely, we have the following criterion.

\section{Lemma 2.1.}

(i) Let $B \in \mathcal{F}_{k+1, m}(\mathrm{Q}, W)$; then $\mathrm{T}(B) \neq \emptyset$.

(ii) Let $B \in \mathcal{F}_{k+1, m}(\mathrm{Q}, W)$ and $U \in \mathrm{T}(B)$; then there is an $L=[H, B]_{k} \in \mathcal{L}^{\alpha}$ such that $U \in L$.

So, if $\mathcal{F}_{k+1, m}(\mathrm{Q}, W) \neq \emptyset$ then $\mathcal{L}^{\alpha} \neq \emptyset$.

(iii) Let $B \in \mathcal{F}_{k+1, m+1}(\mathrm{Q}, W)$; then $\mathrm{T}(B) \neq \emptyset$.

(iv) Let $B \in \mathcal{F}_{k+1, m+1}(\mathrm{Q}, W)$ and $U \in \mathrm{T}(B)$. Then there are:

- an $L^{\prime}=\left[H^{\prime}, B\right]_{k} \in \mathcal{L}^{\omega}$ (provided that $m>0$ ) such that $U \in L^{\prime}$ and

- an $L^{\prime \prime}=\left[H^{\prime \prime}, B\right]_{k} \in \mathcal{A}$ such that $U \in L^{\prime \prime}$.

Consequently, if $\mathcal{F}_{k+1, m+1}(\mathrm{Q}, W) \neq \emptyset$ then $\mathcal{A} \neq \emptyset$, and $\mathcal{L}^{\omega} \neq \emptyset$ when $m>0$.

Proof. To justify (i) present $B$ in the form $B=(B \cap W) \oplus D$, where $D \cap W=\Theta$ and $\operatorname{dim}(D)=k+1-m$. Let $D^{\prime}$ be a $(k-m)$-dimensional subspace of $D$ and put $H:=(B \cap W)+D^{\prime}$. To justify (ii) we simply use (i) with $B$ replaced by $U$ to obtain the subspace $H$.

To justify (iii) we present $B$ in the form $B=(B \cap W) \oplus D$ (now, $\operatorname{dim}(D)=k-m$ ) and proceed analogously to (i): $U=D+Z$, where $Z$ is an $m$-dimensional subspace of $B \cap W$. To justify (iv) to get $H^{\prime}$ we apply (iii) with $B$ replaced by $U$, and to get $H^{\prime \prime}$ we apply (i) with $B$ replaced by $U$. 
Note that, sufficient conditions for the existence of the corresponding subspaces $B$ in Lemma 2.1, i.e. for $\mathcal{F}_{k+1, m}(\mathrm{Q}, W), \mathcal{F}_{k+1, m+1}(\mathrm{Q}, W) \neq \emptyset$ are

$$
m \leq r_{W} \leq r-(k+1)+m \quad \text { and } \quad m+1 \leq r_{W} \leq r-k+m \text {, respectively. }
$$

We say that an $U \in \mathcal{F}_{k, m}(\mathrm{Q}, W)$ is an $\alpha$-point iff each top containing $U$ is of type $\alpha$, i.e. each line through $U$ is of type $\alpha$. Similarly, an $U \in \mathcal{F}_{k, m}(\mathrm{Q}, W)$ is an $\omega$-point iff each top containing $U$ is of type $\omega$, i.e. each line through $U$ is either affine or of type $\omega$.

Lemma 2.2. Let $U \in \mathcal{F}_{k, m}(\mathrm{Q}, W)$.

(i) There is $B$ such that $U \subset B \in \mathcal{F}_{k+1, m}(\mathrm{Q}, W) \cup \mathcal{F}_{k+1, m+1}(\mathrm{Q}, W)$.

(ii) $U$ is an $\alpha$-point iff $U^{\perp} \cap W \subset U$. In this case

$$
w \leq k+m \text {. }
$$

Otherwise, if $U^{\perp} \cap W \not \subset U$ then there is a $B \in \mathcal{F}_{k+1, m+1}(\mathrm{Q}, W)$ such that $U \subset B$.

(iii) $U$ is an $\omega$-point when $U^{\perp} \subset U+W$. In this case

$$
w \geq n+m-2 k .
$$

Otherwise, if $U^{\perp} \not \subset U+W$ then there is a $B \in \mathcal{F}_{k+1, m}(\mathrm{Q}, W)$ such that $U \subset B$.

Proof. Clearly, $U$ is not maximal isotropic, so there is a $B$ such that $U \subset B \in \mathrm{Q}_{k+1}$. As in [12] we obtain $m \leq \operatorname{dim}(B \cap W) \leq m+1$. This justifies (i).

To justify (ii) note that every $B \in \mathrm{Q}_{k+1}$ containing $U$ belongs to $\mathcal{F}_{k+1, m}(\mathrm{Q}, W)$, and then $U \prec B \subset U^{\perp}$ and $U \cap W \subset B \cap W \subset U^{\perp} \cap W$. If we have $\operatorname{dim}(B \cap W)=m$ for all $B$, then $\operatorname{dim}\left(U^{\perp} \cap W\right)=m$ and $U \cap W=U^{\perp} \cap W$. As $U \subset U^{\perp}$ by definition of $U$, the obtained condition is equivalent to $U^{\perp} \cap W \subset U$.

In this case we have $W=(U \cap W) \oplus D$, where $D$ is contained in a linear complement of $U^{\perp} . D$ is at most $\operatorname{codim}\left(U^{\perp}\right)=k$-dimensional, so $\operatorname{dim}(W) \leq m+k$.

To justify (iii) note, first, that if $U \subset B \in \mathrm{Q}_{k+1}$ then $B \in \overline{\mathcal{F}}_{k+1, m+1}(\mathrm{Q}, W)$. So, if $U \prec B \in \mathrm{Q}$, then $B=U \oplus\langle y\rangle$ with $y \in U^{\perp} \backslash U$. If $U^{\perp} \subset U+W$ then $y=u+w$ for some $u \in U$ and $w \in W \backslash U$ and then $B=U \oplus\langle w\rangle$. So, $B \cap W=(U \cap W) \oplus\langle w\rangle$. If there is $y \in U^{\perp} \backslash(U+W)$, then $U+\langle y\rangle$ intersects $W$ in $U \cap W$.

If $U$ is as required above then $n-k=\operatorname{dim}\left(U^{\perp}\right) \leq \operatorname{dim}(U+W)=w+k-m$. This gives $w \geq m+n-2 k$.

From Lemmas 2.1 and 2.2(ii), 2.2(iii) we infer the following geometrical fact.

\section{Corollary 2.3.}

(i) If $w>k+m$ then through each point of $\mathfrak{M}$ there passes an $\omega$-line and an affine line.

(ii) If $w<n+m-2 k$ then through every point of $\mathfrak{M}$ there passes an $\alpha$-line.

Combining Lemmas 2.2(i) with 2.1(ii) and 2.1(iv) we obtain the following Corollary, a weakening of Corollary 2.3 but with more general assumptions.

Corollary 2.4. If $U \in \mathcal{F}_{k, m}(\mathrm{Q}, W)$ then there is a line in $\mathcal{G}_{k, m}(\mathrm{Q}, W)$ through $U$. Consequently, if $\mathcal{F}_{k, m}(\mathrm{Q}, W) \neq \emptyset$, then $\mathcal{G}_{k, m}(\mathrm{Q}, W) \neq \emptyset$. 


\section{Comments to Lemma 2.2.}

ad (ii) Condition (2.1) is a necessary condition for the existence of an $\alpha$-point.

By (1.12) and (2.1) we get $r_{W} \leq r-k+m \leq r-w$ (this implies $r-r_{W} \geq w$ ). This condition is not inconsistent. So, it may happen that $\mathfrak{M}$ contains both $\alpha$ points and $\omega$-tops.

One can note (it is, practically, proved in the proof of Lemma 2.2(ii) that if (2.1) is satisfied and $U \in \mathrm{Q}_{k}$ then there is a subspace $W$ such that $U$ is an $\alpha$-point in $\mathbf{A}_{k, m}(\mathrm{Q}, W)$ and $\operatorname{dim}(W)=w$.

ad (iii) Analogously, condition (2.2) is a necessary condition for the existence of an $\omega$ point.

It is seen that (under suitable assumption, obtained by (1.12) and (2.2): $r-r_{W} \geq$ $k-m \geq n-k-w)$ the space $\mathfrak{M}$ may contain both $\omega$-points and $\alpha$-tops.

And there do exist $W$ for which associated spine spaces contain an $\omega$-point.

As an immediate consequence of Lemma 2.2(iii) we obtain the following.

Corollary 2.5. Assume that $w<n+m+1-2 k$. Then, for every $U \in \mathcal{F}_{k, m+1}(\mathrm{Q}, W)$ there is $L \in \mathcal{A}_{k, m}(\mathrm{Q}, W)$ such that $U=L^{\infty}$.

\section{Examples, particular cases}

Let us examine in some detail polar spine spaces of some, particularly natural classes.

\subsection{Grassmannians of affine polar spaces}

Assume that $W$ is a hyperplane of $\mathfrak{P}$; in turn this is equivalent to say that $\operatorname{Sub}_{1}(W)$ is a hyperplane in $\mathfrak{Q}$. In this case we have

$$
\begin{aligned}
m & =k-1 \text { and } \\
\operatorname{dim}(W \cap Y) & =\left\{\begin{array}{ll}
r & \text { when } Y \subset W \\
r-1 & \text { when } Y \not \subset W
\end{array} \text { for every } Y \in \mathrm{Q}_{r} .\right.
\end{aligned}
$$

It is clear that in this case

$$
\mathcal{F}_{k, m}(\mathrm{Q}, W) \neq \emptyset \text {; in view of Corollary } 2.4, \mathbf{A}_{k, m}(\mathrm{Q}, W) \text { is nontrivial }
$$

simply, because it is impossible to have $\mathrm{Q}_{k} \subset \operatorname{Sub}_{k}(W)$. However, this case raises several degenerations concerning the structure of strong subspaces of $\mathfrak{M}$.

\section{Lemma 3.1.}

(i) Let $B \in \operatorname{Sub}_{k+1}(\mathbb{V})$. Then either $\operatorname{dim}(B \cap W)=k+1=m+2$ (and then $B \subset W$ ) or $\operatorname{dim}(B \cap W)=k=m+1$. Therefore, there is no strong subspace in $\mathcal{T}^{\alpha}$. Moreover, by the same reasons, $\mathcal{L}^{\alpha}=\emptyset$.

(ii) If $B \in \mathcal{F}_{k+1, m+1}(\mathrm{Q}, W)$ then $\mathrm{T}(B) \cap \mathcal{F}_{k, m}(\mathrm{Q}, W) \in \mathcal{T}^{\omega}$ is a $k$-dimensional punctured projective space. 
(iii) Let $X=[H, Y]_{k} \cap \mathcal{F}_{k, m}(\mathrm{Q}, W), H \in \operatorname{Sub}_{k-1}(\mathbb{V}), Y \in \mathrm{Q} r$. Assume that $\operatorname{dim}(H \cap W)=m=k-1$ i.e. $H \subset W$. If $Y \subset W$ then, clearly, $X=\emptyset$. If $Y \not \subset W$ then $X \in \mathcal{S}^{\alpha}$ is a $(r-k)$-dimensional affine space.

(iv) Let $X=[H, Y]_{k} \cap \mathcal{F}_{k, m}(\mathrm{Q}, W), H \in \operatorname{Sub}_{k-1}(\mathbb{V}), Y \in \mathrm{Q} r$. Assume that $\operatorname{dim}(H \cap W)=m-1=k-2$ i.e. $H \not \subset W$. Then $Y \not \subset W$ and, consequently, $\operatorname{dim}(Y \cap W)=r-1$. In this case $X \in \mathcal{S}^{\omega}$ is a $(r-k)$-dimensional projective space.

Corollary 3.2. If $4 \leq k+2 \leq r$ then every line of $\mathfrak{M}$ has at least two extensions to a maximal at least 2-dimensional strong subspace: one to a top, and one to a star.

\subsection{Spine spaces with isotropic 'holes'}

Next, let us assume that $W \in \mathrm{Q}$ i.e. $W$ is isotropic. In this case we have

$$
r_{W}=w .
$$

So, let $m<w, k$; let us take arbitrary $D \in \operatorname{Sub}_{m}(W)$ and $Y \in \mathrm{Q}_{r}$ with $W \subset Y$. Then there is $Y_{0} \in \mathrm{Q}_{r}$ such that $Y \cap Y_{0}=D$. Consider any $U$ such that $\operatorname{dim}(U)=k$ and $D \subset U \subset Y_{0}$; then $U \in \mathcal{F}_{k, m}(\mathrm{Q}, W)$. Thus we have proved that

$$
\mathcal{F}_{k, m}(\mathrm{Q}, W) \neq \emptyset \text {; in view of Corollary } 2.4, \mathbf{A}_{k, m}(\mathrm{Q}, W) \text { is nontrivial. }
$$

Note that if we assume (1.10) then $k+w-m \leq r+r-m \leq n-m \leq n$ follows, so (1.8) holds as well.

Next, let us pay attention to the problem of extending lines. Namely, let $L=\mathbf{p}(H, B) \in$ $\mathcal{L}^{\omega}$. So, $\operatorname{dim}(B \cap W)=m+1$. Suppose that $r=m+1$; then we obtain contradictory $m<k<r=m+1$. As above, we extend $W$ to a maximal isotropic $Y$ and find maximal isotropic $Y^{\prime}$ with $Y \cap Y^{\prime}=B$. This proves

Lemma 3.3. If $k<r-1$, then every line in $\mathcal{L}^{\omega}$ can be extended to an at least 2 -dimensional star.

\section{Binary collinearity}

Let us start with a Chow'like result concerning binary collinearity $\boldsymbol{\lambda}$ of points in a polar spine space $\mathfrak{M}=\mathbf{A}_{k, m}(\mathrm{Q}, W)$ defined for some integers $k, m$ and a fixed subspace $W$ of a vector space $V$ equipped with a suitable form $\xi$. To this aim standard reasoning similar to this of $[6,7,17]$ can be used:

a line through two distinct points is the intersection of all the maximal $\lambda$-cliques which contain these points.

In the sequel we intensively analyse Table 2. Let $U_{1} \lambda U_{2}, U_{1} \neq U_{2}$. Put $L=\overline{U_{1}, U_{2}}$. Evidently, every line $L=\mathbf{p}(H, B)$ can be extended to a top $T=\mathrm{T}(B) \cap \mathcal{F}_{k, m}(\mathrm{Q}, W)$, which is a $(k-m)$-dimensional $\left(T \in \mathcal{T}^{\alpha}\right)$ or a $k$-dimensional $\left(T \in \mathcal{T}^{\omega}\right)$ slit space. We have assumed that $k>1$. So, when $m<k-1$ then $T$ is greater than $L$. For any triangle $U_{1}, U_{2}, U_{3} \in T$ we have $\boldsymbol{\Delta}_{\boldsymbol{\lambda}}\left(U_{1}, U_{2}, U_{3}\right)$ and $T=\left[U_{1}, U_{2}, U_{3}\right]_{\boldsymbol{\lambda}}$.

If $L$ is an $\alpha$-projective line or an affine line then it has at least one extension to a star $S$ in $\mathcal{S}^{\alpha}$, which are $(r-k)$-dimensional slit spaces. Consequently, $L=T \cap S$. Assume that $k<r-1$, so $L \subsetneq S$. 
In this point we can choose one of the following two ways. Firstly, we notice that there is a finite system $U_{1}, U_{2}, \ldots, U_{t} \in S$ such that $\boldsymbol{\Delta}_{\boldsymbol{\lambda}}\left(U_{1}, U_{2}, \ldots, U_{t}\right)$, so $S^{\prime}:=S=$ $\left[U_{1}, U_{2}, \ldots, U_{t}\right]_{\lambda}$. Secondly, we can extend $U_{1}, U_{2}$ to any triangle $U_{1}, U_{2}, U_{3} \in S$ and note that $S^{\prime}:=\left[U_{1}, U_{2}, U_{3}\right]_{\lambda}$ is the union of all the extensions of the plane spanned by $U_{1}, U_{2}, U_{3}$ to a maximal $\lambda$-clique. In both cases $L=S^{\prime} \cap T$ and thus $L$ can be defined in terms of $\boldsymbol{\lambda}$.

A problem may arise when $L \in \mathcal{L}^{\omega}$. In this case each extension of $L$ to a star $S$ is contained in a segment $[H, Y]_{k}$ with a maximal totally isotropic extension $Y$ of $B \supset$ $H$ and it has dimension $\operatorname{dim}(W \cap Y)-m$. So, it may degenerate to the line $L$ when $\operatorname{dim}(W \cap Y)=m+1$. Is it possible that every such an extension $Y$ intersects $W$ in dimension $m+1$ ? Recall that the condition $L \in \mathcal{L}^{\omega}$ yields $\operatorname{dim}(B \cap W)=m+1$ and therefore we obtain $W \cap Y=W \cap B$, for every $\mathrm{Q}_{r} \ni Y \supset B$. So, our problematic case reduces to the question: for which $B \in \mathcal{F}_{k+1, m+1}(\mathrm{Q}, W)$ there is no reasonable extension $Y$ and: when each such a $B$ has a required extension. Note that to find $Y$ it suffices to find $D$ such that $B \prec D \in \mathrm{Q}$ and $\operatorname{dim}(D \cap W)=m+2$; then $Y$ is an extension of $D$ to a maximal totally isotropic subspace. On the other hand, the existence of $D$ in question can be assured by a suitable substitution in Lemma 2.2(ii), which yields a sufficient condition for the existence of our $Y$ :

$$
w>k+m+2 .
$$

As a consequence we can formulate the following result.

Theorem 4.1 (The Chow Theorem for $\mathfrak{M})$. If $m<k-1, k<r-1$, and each line in $\mathcal{L}^{\omega}$ can be extended to at least 2-dimensional star (which is assured, e.g. by (4.1)) then the structures $\mathfrak{M}$ and $\left\langle\mathcal{F}_{k, m}(\mathrm{Q}, W), \boldsymbol{\lambda}\right\rangle$ are definitionally equivalent.

In particular, in view of Corollary 3.2 and Lemma 3.3, the Chow theorem holds in $\mathfrak{M}$ when $W$ is an isotropic subspace and $k<r-1$, and it holds in $\mathfrak{M}$ when $W$ is a hyperplane and $4 \leq k+2 \leq r$.

One can continue these investigations in the fashion of [17] considering graphs of collinearity with some sorts of lines distinguished $\left(\boldsymbol{\lambda}^{\alpha}, \boldsymbol{\lambda}^{\omega}, \boldsymbol{\lambda}^{\alpha \vee \omega}\right.$ etc.). Observing criteria in Lemma 2.2 and Corollary 2.5 we see that it may be a hard work: $\alpha$-points and $\omega$-points may appear, 'deep' improper points may appear as well.

\section{Maximal cliques of $\lambda^{\sigma}$}

Let $\sigma$ be a one of the symbols

$$
\alpha, \omega, \alpha \vee \omega, \alpha^{+}, \omega^{+} \text {. }
$$

The classes $\mathcal{L}^{\sigma}$ with $\sigma \in\{\alpha, \omega\}$ are already defined (usually, the arguments like $k, m$, $\mathbb{V}, \mathrm{Q}, W$ will be omitted, if unnecessary or fixed). Next, $\mathcal{L}^{\sigma^{+}}:=\mathcal{L}^{\sigma} \cup \mathcal{A}$, and, finally $\mathcal{L}^{\alpha \vee \omega}=\mathcal{L}^{\alpha} \cup \mathcal{L}^{\omega}$. It is evident that

$$
\mathfrak{M}^{\sigma}:=\left\langle\mathcal{F}_{k, m}(\mathrm{Q}, W), \mathcal{L}_{k, m}^{\sigma}(\mathrm{Q}, W)\right\rangle
$$

is a partial linear space for every admissible symbol $\sigma$ as above, but it may be trivial for particular values of $k, m, r, w$ etc.: it may have a void line set. Let us write $\lambda^{\sigma}$ for the binary collinearity of points of $\mathfrak{M}^{\sigma}$. Let $\lambda^{\text {af }}$ be the binary collinearity in

$$
\mathfrak{A}=\left\langle\mathcal{F}_{k, m}(\mathrm{Q}, W), \mathcal{A}_{k, m}, \|\right\rangle .
$$


In the first part of this section we shall determine (maximal) cliques of $\boldsymbol{\lambda}^{\sigma}$ for particular values of $\sigma$ as above. Clearly, each such a clique is a $\boldsymbol{\lambda}$-clique. So, it is contained in an appropriate strong subspace of $\mathfrak{M}$.

We begin with some results which state, generally, that the affine lines in many cases can be 'eliminated': they are definable in terms of other projective lines.

Proposition 5.1. Assume that $m>0$ or $w<r-k$. Then for arbitrary triple $U_{1}, U_{2}, U_{3} \in$ $\mathcal{F}_{k, m}(\mathrm{Q}, W)$ we have

$$
\begin{aligned}
& \text { there is a line } L_{0} \in \mathcal{A} \text { s.t. } U_{1}, U_{2}, U_{3} \in L_{0} \Longleftrightarrow \\
& \text { there is a triangle } L_{1}, L_{2}, L_{3} \in \mathcal{L}_{k, m}^{\alpha \vee \omega} \text { s.t. } U_{i} \in L_{i} \text { for } i=1,2,3 \\
& \& \text { there is no } L \in \mathcal{L}^{\alpha \vee \omega} \text { s.t. } U_{i}, U_{j} \in L \text { for some } 1 \leq i<j \leq 3 .
\end{aligned}
$$

Proof. Let $U_{1}, U_{2}, U_{3} \in L_{0} \in \mathcal{A}$; then we can write $L_{0}=\mathbf{p}(H, B) \cap \mathcal{F}_{k, m}(\mathrm{Q}, W)$ for suitable $H, B$. As in the proof of Lemma 6.6 we examine extensions of $L_{0}$ to maximal strong subspaces of $\mathfrak{M}$. First, let us have a look at $\mathrm{T}(B) \cap \mathcal{F}_{k, m}(\mathrm{Q}, W)$. It is an affine space only when $m=0$; otherwise it contains a nonaffine semiaffine plane $A$ which contains $L_{0}$. The lines on $A$ are all in $\mathcal{L}^{\alpha \vee \omega}$ except the direction of $L_{0}$. It suffices to find adequate triangle on $A$ to justify ( $\Rightarrow$ :) of (5.2).

Next, assume that $m=0$ and take a look at extensions of $L_{0}$ of the form $[H, Y]_{k} \cap$ $\mathcal{F}_{k, m}(\mathrm{Q}, W)$, then $B \subset Y \in \mathrm{Q}_{r}$. This extension is an affine space when $\operatorname{dim}(W \cap Y)=$ $r-k$. If there is no such $Y$, which is assured by the condition assumed, our extension contains a plane $A$ as above and ( $\Rightarrow$ :) of (5.2) is justified.

To prove $(\Leftarrow:)$ it suffices to note that a triangle spans a plane $A$ in $\mathfrak{M}$. Since this plane contains projective lines it is not affine, and since there are non projectively joinable points on $A$ it contains just one direction of affine lines. The rest is evident.

Thus we have proved the following result.

Proposition 5.2. Under assumptions made in Proposition 5.1 the class $\mathcal{A}_{k, m}(\mathrm{Q}, W)$ is definable in $\mathfrak{M}^{\alpha \vee \omega}$. That means: $\mathfrak{M}$ is definable in $\mathfrak{M}^{\alpha \vee \omega}$.

Remark 5.3. Analysing the proof of Proposition 5.1 one can note an even more detailed result:

(i) If $m>0$ then $\mathcal{A}$ is definable in $\mathfrak{M}^{\omega}$ and therefore then $\mathfrak{M}^{\omega^{+}}$is definable in $\mathfrak{M}^{\omega}$.

(ii) If every affine line $L=\mathbf{p}(H, B)$ can be extended to a non-affine $\operatorname{star}(\operatorname{dim}(W \cap Y) \geq$ $r-k+m-3$ for some maximal isotropic $Y$ containing $B$ ) then $\mathcal{A}$ is definable in $\mathfrak{M}^{\alpha}$. So, $\mathfrak{M}^{\alpha^{+}}$is definable in $\mathfrak{M}^{\alpha}$.

For an arbitrary set $X$ of points we write

$$
\mathrm{L}(X)=\left\{L \in \mathcal{G}_{k, m}(\mathrm{Q}, W): L \subset X\right\} .
$$

Let us remind well known and fundamental classification of lines in strong subspaces of $\mathfrak{M}$.

Fact 5.4. Let $X$ be a strong subspace of $\mathfrak{M}$ and $\mathcal{X}=\mathrm{L}(X)$.

$$
\begin{array}{ll}
\text { If } X \in \mathcal{T}^{\alpha} \text { then } \mathcal{X} \subset \mathcal{L}^{\alpha}, & \text { if } X \in \mathcal{S}^{\alpha} \text { then } \mathcal{X} \subset \mathcal{L}^{\alpha^{+}}, \\
\text {if } X \in \mathcal{T}^{\omega} \text { then } \mathcal{X} \subset \mathcal{L}^{\omega^{+}}, & \text {if } X \in \mathcal{S}^{\omega} \text { then } \mathcal{X} \subset \mathcal{L}^{\omega} .
\end{array}
$$


Let us note an elementary

Fact 5.5. Let $\mathfrak{S}$ be a $n_{0}$-dimensional slit space with a $w_{0}$-dimensional hole i.e. let $\mathfrak{S}$ result from a $n_{0}$-dimensional projective space by deleting a $w_{0}$-dimensional subspace $\mathcal{D}$. Let $\mathcal{L}_{0}$ be the class of projective lines of $\mathfrak{S}$ and $\lambda_{0}$ be the binary collinearity determined by $\mathcal{L}_{0}$. Then

(i) The maximal affine subspaces of $\mathfrak{S}$ (i.e. maximal strong subspace w.r.t. to the family of affine lines of S) are $w_{0}+1$ dimensional affine spaces. Two such subspaces either coincide or are disjoint.

(ii) The maximal projective subspaces of $\mathfrak{S}$ are $\left(n_{0}-w_{0}-1\right)$-dimensional projective spaces. These are linear complements of $D$ and the elements of $\mathcal{K}^{*}\left(\boldsymbol{\lambda}_{0}\right)$.

(iii) Let $X$ be a maximal projective subspace of $\mathfrak{S}$; then $X \in \mathcal{K}_{\boldsymbol{\lambda}_{0}}^{n_{0}-w_{0}}$.

If $w_{0} \leq n_{0}-3$ (i.e. every projective line of $\mathfrak{S}$ has two distinct extensions to maximal projective subspaces) then the Chow Theorem holds:

The class $\mathcal{L}_{0}$ is definable in terms of $\boldsymbol{\lambda}_{0}$.

Observing Table 2 and Fact 5.5 we conclude with the following.

\section{Corollary 5.6.}

(i) The maximal $\boldsymbol{\lambda}^{\alpha}$-cliques are $(k-m)$-dimensional projective tops: elements of $\mathcal{T}^{\alpha}$, and $(r+m-k-\operatorname{dim}(W \cap Y))$-dimensional projective spaces of the form

$$
[H, E]_{k} \text {, where } H \subset E \subset Y, E \cap((W \cap Y)+H)=H
$$

contained in a suitable element $[H, Y]_{k} \cap \mathcal{F}_{k, m}(\mathrm{Q}, W)$ of $\mathcal{S}^{\alpha}$.

(ii) The maximal $\boldsymbol{\lambda}^{\omega}$-cliques are $(\operatorname{dim}(W \cap Y)-m)$-dimensional projective stars: elements of $\mathcal{S}^{\omega}$, and m-dimensional projective spaces of the form

$$
[G, B]_{k} \text {, where } G \subset B, G \cap(B \cap W)=\Theta
$$

contained in a suitable element $\mathrm{T}(B) \cap \mathcal{F}_{k, m}(\mathrm{Q}, W)$ of $\mathcal{T}^{\omega}$.

(iii) The maximal $\lambda^{\alpha^{+}}$-cliques are elements of $\mathcal{T}^{\alpha} \cup \mathcal{S}^{\alpha}$, and the maximal $\lambda^{\omega^{+}}$-cliques are elements of $\mathcal{T}^{\omega} \cup \mathcal{S}^{\omega}$.

(iv) $\mathcal{K}^{*}\left(\boldsymbol{\lambda}^{\alpha \vee \omega}\right)=\mathcal{K}^{*}\left(\boldsymbol{\lambda}^{\alpha}\right) \cup \mathcal{K}^{*}\left(\boldsymbol{\lambda}^{\omega}\right)$, so the maximal $\boldsymbol{\lambda}^{\alpha \vee \omega}$-cliques are of the form (i) and of the form (ii) above.

Corollary 5.7. The following variants of the Chow Theorem hold in projective reducts of $\mathfrak{M}$.

(i) If $m>1$ then $\mathfrak{M}^{\omega}$ is definable in $\left\langle\mathcal{F}_{k, m}(\mathrm{Q}, W), \lambda^{\omega}\right\rangle$.

(ii) If every projective line $L=\mathbf{p}(H, B) \in \mathcal{L}^{\alpha}$ can be extended to a non-affine star $(\operatorname{dim}(W \cap Y) \leq r-k+m-2$ for some maximal isotropic $Y$ containing $B)$ then $\mathfrak{M}^{\alpha}$ is definable in $\left\langle\mathcal{F}_{k, m}(\mathrm{Q}, W), \boldsymbol{\lambda}^{\alpha}\right\rangle$. 


\section{Parallelism, horizon, projective completion(s)}

Let us summarize the following

(i) $\left\{L^{\infty}: L \in \mathcal{A}_{k, m}\right\} \subset \mathcal{F}_{k, m+1}(\mathrm{Q}, W)$.

(ii) by Lemma 2.2(ii) $\left\{L^{\infty}: L \in \mathcal{A}_{k, m}\right\} \supset\left\{U \in \mathcal{F}_{k, m+1}(\mathrm{Q}, W): U^{\perp} \cap W \not \subset U\right\}$,

(iii) $\left\{L^{\infty}: L \in \mathcal{A}_{k, m}\right\} \supset \mathcal{F}_{k, m+1}(\mathrm{Q}, W)$, when $w<n+m+1-2 k$ by Corollary 2.5 .

Note 6.1. The set $\left\{L^{\infty}: L \in \mathcal{A}_{k, m}\right\}$ will be frequently referred to as the horizon of $\mathfrak{M}$. We warn that, generally it does not coincide with the horizon $\mathrm{Q}_{k} \backslash \mathcal{F}_{k, m}(\mathrm{Q}, W)$ as defined in Section 1.

Note that the inequality in (iii) above is only sufficient. One can compute e.g.

Lemma 6.2. Let $W \in \mathrm{Q}$. Then the claim of Corollary 2.5 holds i.e. for every $U \in$ $\mathcal{F}_{k, m+1}(\mathrm{Q}, W)$ there is an $L \in \mathcal{A}_{k, m}(\mathrm{Q}, W)$ such that $U=L^{\infty}$. Consequently,

$$
\left\{L^{\infty}: L \in \mathcal{A}_{k, m}\right\}=\mathcal{F}_{k, m+1}(\mathrm{Q}, W) .
$$

Proof. By assumption, $\operatorname{dim}(U \cap W)=m+1$. There are extensions $Y_{1}, Y_{2} \in \mathrm{Q}_{r}$ such that $U \subset Y_{1}, W \subset Y_{2}$, and $Y_{1} \cap Y_{2}=U \cap W$. Take $B \in\left[U, Y_{1}\right]_{k+1}$; then $B \in$ $\mathcal{F}_{k+1, m+1}(\mathrm{Q}, W)$ and we are through.

For a subset $X$ of $\mathcal{F}_{k, m}(\mathrm{Q}, W)$ we write

$$
X^{\infty}:=\left\{N^{\infty}: \mathcal{A}_{k, m} \ni N \subset X\right\} .
$$

Lemma 6.3. Let $L=\mathbf{p}(H, B) \in \mathcal{L}_{k, m+1}^{\omega} \cup \mathcal{L}_{k, m+1}^{\alpha}$.

(i) If $L \in \mathcal{L}^{\alpha}$ then there is in $\mathfrak{M}$ a plane $A=[G, B]_{k} \cap \mathcal{F}_{k, m}(\mathrm{Q}, W)$ with $G \in$ $\mathcal{F}_{k-2, m}(\mathrm{Q}, W)$ such that $A^{\infty}=L$.

(ii) Assume that $w<n+m-2 k$. If $L \in \mathcal{L}^{\omega}$ then $A=[H, E]_{k} \cap \mathcal{F}_{k, m}(\mathrm{Q}, W)$ with some $E \in \mathcal{F}_{k+2, m+2}(\mathrm{Q}, W)$ is a plane in $\mathfrak{M}$ such that $A^{\infty}=L$.

Proof. Ad (i): By assumption, $B \in \mathcal{F}_{k+1, m+1}(\mathrm{Q}, W)$ and $H \in \mathcal{F}_{k-1, m+1}(\mathrm{Q}, W)$. There is a point $U \in L$, so $U \in \mathcal{F}_{k, m+1}(\mathrm{Q}, W)$. By Lemma 2.1(iii) there is an $H_{0}$ such that $U \succ H_{0} \in \mathcal{F}_{k-1, m}(\mathrm{Q}, W)$. Set $G=H_{0} \cap H$; clearly, $\operatorname{dim}(G)=k-2$, so $[G, B]_{k}$ is a plane in $\mathbf{P}_{k}(\mathrm{Q})$. Taking into account the fact that $H, H_{0} \succ G$ we obtain $\operatorname{dim}(G \cap W) \in$ $\{m+1, m\}$ and $\operatorname{dim}(G \cap W) \in\{m, m-1\}$. Thus $\operatorname{dim}(G \cap W)=m$. As $L \subset[G, B]_{k}$ and $[G, B]_{k} \supset\left[H_{0}, B\right]_{k}$ while $\left[H_{0}, B\right]_{k} \cap \mathcal{F}_{k, m}(\mathrm{Q}, W) \in \mathcal{A}_{k, m}$ we get that $A \cap \mathcal{F}_{k, m}(\mathrm{Q}, W)$ is a plane in $\mathfrak{M}$ with $A^{\infty}=L$.

Ad (ii): By assumption, $B \in \mathcal{F}_{k+1, m+2}(\mathrm{Q}, W)$ and $H \in \mathcal{F}_{k-1, m}(\mathrm{Q}, W)$. As above, we take any $U \in L$, so $U \in \mathcal{F}_{k, m+1}(\mathrm{Q}, W)$. By assumption of (ii) (they yield $w<n+$ $(m+2)-2(k+1))$ and Lemma 2.2(iii) there is an $E$ such that $B \prec E \in \mathcal{F}_{k+2, m+2}(\mathrm{Q}, W)$. Next, there is $B_{0} \in \mathcal{F}_{k+1, m+1}(\mathrm{Q}, W)$ with $U \subset E: B=U+\langle b\rangle$ with a $b \in W$ and $E=B+\langle e\rangle$ with an $e \notin W$; we take $B_{0}=U+\langle e\rangle$. Clearly, $E=B+B_{0}$ and $\left[H, B_{0}\right]_{k} \cap \mathcal{F}_{k, m}(\mathrm{Q}, W) \in \mathcal{A}_{k, m}$. As above we argue that $A=[H, E]_{k} \cap \mathcal{F}_{k, m}(\mathrm{Q}, W)$ is a plane in $\mathfrak{M}$, and $L=A^{\infty}$. 
Roughly speaking, Lemma 6.3 gives sufficient condition under which a (projective) line $L$ of $\mathbf{A}_{k, m+1}(\mathrm{Q}, W)$ can be considered as a 'horizon' - the set of improper points of a plane in $\mathbf{A}_{k, m}(\mathrm{Q}, W)$. On the other hand, considering classification of planes in $\mathbf{A}_{k, m}(\mathbb{V}, W)$ presented in some details in [14] we easily conclude with the following

Lemma 6.4. Let $X \subset \operatorname{Sub}_{k}(\mathbb{V})$ and $A=X \cap \mathcal{F}_{k, m}(\mathrm{Q}, W)$ be a plane of $\mathfrak{M}$ such that $A^{\infty}$ is a line of $\mathbf{A}_{k, m+1}(\mathrm{Q}, W)$. Then one of the following holds:

(i) $X=[G, B]_{k}$ for some $G \in \mathcal{F}_{k-2, m}(\mathrm{Q}, W), B \in \mathcal{F}_{k+1, m+1}(\mathrm{Q}, W)$.

(ii) $X=[H, E]_{k}$ for some $H \in \mathcal{F}_{k-1, m}(\mathrm{Q}, W)$ and $E \in \mathcal{F}_{k+2, m+2}(\mathrm{Q}, W)$.

Conversely, if $X$ is defined by (i) then $X \cap \mathcal{F}_{k, m+1}(\mathrm{Q}, W)=\left(X \cap \mathcal{F}_{k, m}(\mathrm{Q}, W)\right)^{\infty} \in$ $\mathcal{L}_{k, m+1}^{\alpha}$, and if (ii) holds, then $X \cap \mathcal{F}_{k, m+1}(\mathrm{Q}, W) \in \mathcal{L}_{k, m+1}^{\omega}$.

So, Lemma 6.4 states that the 'horizon' of any (affine) plane of $\mathcal{F}_{k, m}(\mathrm{Q}, W)$ is a (projective) line of $\mathcal{F}_{k, m+1}(\mathrm{Q}, W)$. As usually, the conditions of Lemma 6.3 are only sufficient. Dealing with concrete cases one should look for suitable extendability more or less 'by hand'. Let us quote an example:

Lemma 6.5. Let $W \in \mathrm{Q}$. If $L=\mathbf{p}(H, B) \in \mathcal{L}_{k, m+1}^{\omega}$ then $A=[H, E]_{k} \cap \mathcal{F}_{k, m}(\mathrm{Q}, W)$ with some $E \in \mathcal{F}_{k+2, m+2}(\mathrm{Q}, W)$ is a plane in $\mathfrak{M}$ such that $A^{\infty}=L$.

Hint. With the reasoning as in the proof of Lemma 6.3(ii) we look for an $E$ such that $B \prec E \in \mathcal{F}_{k+2, m+2}(\mathrm{Q}, W)$. It suffices to find an $E$ such that $E \cap W=B \cap W$ just considering suitable maximal isotropic extensions of $B$ and $W$.

To accomplish this part of investigations on the parallelism let us check if directions are 'isolated': when for an affine line $L$ of $\mathfrak{M}$ there are other lines parallel to $L$ and coplanar with $L$; with the plane in question being affine in $\mathfrak{M}$.

Lemma 6.6. Let $L=\mathbf{p}(H, B) \in \mathcal{A}_{k, m}$ and $U=L^{\infty}$.

(i) Assume that $k>m+1$. There is an $L_{0}=\mathbf{p}\left(H_{0}, B\right) \in \mathcal{L}_{k, m+1}^{\alpha}$ such that $U \in L_{0}$ and $A=\left[H_{0} \cap H, B\right]_{k} \cap \mathcal{F}_{k, m}(\mathrm{Q}, W)$ is a plane in $\mathfrak{M}$ such that $A^{\infty}=L_{0}$. We have $\operatorname{dim}\left(\left(H_{0} \cap H\right) \cap W\right)=m-1$.

(ii) If $B$ has an extension to a $Y \in \mathrm{Q}_{r}$ such that $\operatorname{dim}(W \cap Y) \geq m+2$ (this yields, necessarily, $\left.m+2 \leq r_{W}\right)$ then there exists an $L_{1}=\mathbf{p}\left(H, B_{1}\right) \in \mathcal{L}_{k, m+1}^{\omega}$ such that $U \in L_{1}$ and $A=\left[H, B+B_{1}\right]_{k}$ is a plane in $\mathfrak{M}$ such that $A^{\infty}=L_{1}$. We have $\operatorname{dim}\left(\left(B_{1}+B\right) \cap W\right)=m+2$.

Proof. Let us begin with a reminder: $H \in \mathcal{F}_{k-1, m}(\mathrm{Q}, W), B \in \mathcal{F}_{k+1, m+1}(\mathrm{Q}, W)$. Have a look at the extension of $L$ to a top $T=\mathrm{T}(B) \cap \mathcal{F}_{k, m}(\mathrm{Q}, W)$ (an $\omega$-top in this case). Since $k>m+1$, this is a semiaffine space, and its hole is at least 1-dimensional. Let $L_{0}$ be any line of $\mathbf{P}_{k}(\mathbb{V})$ contained in this hole and $A$ be the plane spanned by $L \cup L_{0}$. That way we justify (i).

Next, let us look for appropriate extension of $L$ to an $\alpha$-star $S=[H, Y]_{k} \cap \mathcal{F}_{k, m}(\mathrm{Q}, W)$. In general, it is a $(r-k)$-dimensional semiaffine space. Since $\mathrm{Q}_{k+1} \neq \emptyset$ we have $k+1 \leq r$. So, $S$ is at least a line. To assure that the hole of $S$ contains at least a line of $\mathbf{P}_{k}(\mathbb{V})$ we must assume that $\operatorname{dim}(W \cap Y) \geq m+2$. That way we justify (ii). 
Let us remind that for distinct affine lines $L_{1}, L_{2}$ contained in a strong subspace of $\mathbf{A}_{k, m}(\mathbb{V}, W)$ their parallelism $\|$ can be characterized by the following formula (so called Veblenian parallelism).

$$
\begin{aligned}
& L_{1} \|_{\mathrm{v}} L_{2} \Longleftrightarrow \text { there are lines } L_{1}^{\prime}, L_{2}^{\prime} \text { s.t. }\left|L_{1}^{\prime} \cap L_{2}^{\prime}\right|=1, \\
& \qquad \begin{array}{l}
\text { and } L_{1}^{\prime} \cap L_{2}^{\prime} \cap L_{i}=\emptyset,\left|L_{i}^{\prime} \cap L_{j}\right|=1 \text { for } i=1,2,
\end{array}
\end{aligned}
$$

and then $L_{1} \| L_{2}$ iff $L_{1} \|_{\mathrm{v}} L_{2}$. It is easy to note that the same formula (6.1) characterizes parallelism of affine lines contained in a common strong subspace of $\mathfrak{M}$.

Let us begin with a special form of connectedness of the space of lines over $\mathfrak{M}$ :

Lemma 6.7. Let $U \in \mathcal{F}_{k, m+1}(\mathrm{Q}, W)$ and $L_{1}^{\infty}=U=L_{2}^{\infty}$ for $L_{1}, L_{2} \in \mathcal{A}_{k, m}$. Moreover, assume that $k \leq r-2$. Then there are lines $M_{1}, \ldots, M_{t} \in \mathcal{A}_{k, m}(t \leq r+1)$ such that $L_{1}=M_{1}, L_{2}=M_{t}$, and $M_{i}^{\infty}=U, M_{i}, M_{i+1}$ are in a strong (semiaffine) subspace of $\mathfrak{M}$ or $M_{i}=M_{i+1}$, for $i=1, \ldots, t-1$.

Proof. Write $M_{1}:=L_{1}$. We have $H_{1}, H_{2} \subset U \subset B_{1}, B_{2}, U \in \mathcal{F}_{k, m+1}(\mathrm{Q}, W)$ and $B_{i} \in$ $\mathcal{F}_{k+1, m+1}(\mathrm{Q}, W), H_{i} \in \mathcal{F}_{k-1, m}(\mathrm{Q}, W)$ for $i=1,2$. Put $N_{1}:=\left[H_{1}, B_{2}\right]_{k} \cap \mathcal{F}_{k, m}(\mathrm{Q}, W)$, $N_{2}:=\left[H_{2}, B_{1}\right]_{k} \cap \mathcal{F}_{k, m}(\mathrm{Q}, W)$. Then $N_{1}, N_{2} \in \mathcal{A}_{k, m}, N_{2}^{\infty}=U=N_{1}^{\infty}$.

If $L_{1}=N_{2}$ we set $M_{2}:=L_{1}$. Assume that $L_{1} \neq N_{2}$. Note that $L_{1}, N_{2} \in \mathrm{T}\left(B_{1}\right) \cap$ $\mathcal{F}_{k, m}(\mathrm{Q}, W) \in \mathcal{T}^{\omega}$. So, we set $M_{2}:=N_{2}$.

Observe that $N_{2}, L_{2} \subset\left[H_{2}, V\right]_{k} \cap \mathcal{F}_{k, m}(\mathrm{Q}, W)$. So, the problem reduces to find a required sequence of lines in the projective star $\mathrm{S}\left(H_{2}\right)$. Let $B_{1} \subset Y^{\prime} \in \mathrm{Q}_{r}, B_{2} \subset Y^{\prime \prime} \in$ $\mathrm{Q}_{r}$. There is a sequence $Y_{2}, \ldots, Y_{t}$ of elements of $\mathrm{Q}_{r}$ such that $Y^{\prime}=Y_{2}, Y^{\prime \prime}=Y_{t}$, and $U \subset Y_{i}, E_{i}:=Y_{i} \cap Y_{i+1}, \operatorname{dim}\left(E_{i}\right)=r-1$ for $i=2, \ldots, t-1, t \leq r+1$. Then $\operatorname{dim}\left(E_{i} \cap W\right) \geq m+1$. From our assumption $k+1 \leq r-1=\operatorname{dim}\left(E_{i}\right)$. So, for every $i=3, \ldots, t-1$ one can find $D_{i}$ such that $U \prec D_{i} \subset E_{i-1}$ and $\operatorname{dim}\left(D_{i} \cap W\right)=m+1$. With $N_{i}=\left[H_{2}, D_{i}\right]_{k}$ we close our proof.

Corollary 6.8. Under assumptions of Lemma 6.7 the parallelism $\|$ in $\mathfrak{M}$ coincides with the transitive closure of $\|_{\mathrm{v}}$. Actually, it is the $(r+1)$-th relational power $\underbrace{\left\|_{\mathrm{v}} \circ \cdots \circ\right\|_{\mathrm{v}}}_{(r+1) \text { times }}$ of $\|_{\mathrm{v}}$, defined by (6.1), and therefore $\|$ is definable in the incidence structure $\mathfrak{M}$.

As an immediate corollary we conclude with the following theorem.

Theorem 6.9. Assume the following

(1) $w<n+m-2 k$ to assure that every line in $\mathcal{L}_{k, m+1}^{\omega}$ can be extended to a nontrivial $\alpha$-star of $\mathfrak{M}$ (cf. Lemma 6.3),

(2) $w<n+m+1-2 k$ to assure extendability of each improper point to an affine line (cf. Corollary 2.5),

(3) $m+1>0$ or $w<r-k$ to assure definability of $\mathcal{A}_{k, m+1}$ in $\mathbf{A}_{k, m+1}(\mathrm{Q}, W)$ in terms of its projective lines (cf. Proposition 5.2),

(4) $k \leq r-2$, to assure definability of parallelism in $\mathfrak{M}$ (cf. Corollary 6.8).

Then $\mathbf{A}_{k, m+1}(\mathrm{Q}, W)$ is definable within $\mathbf{A}_{k, m}(\mathrm{Q}, W)$. 
In analogy to [17] in the fragment of $\mathbf{P}_{k}(\mathrm{Q})$ determined by $\mathcal{R}:=\mathcal{F}_{k, m}(\mathrm{Q}, W) \cup$ $\mathcal{F}_{k, m+1}(\mathrm{Q}, W)$ (i.e. the points of $\mathfrak{M}$ and the points of the "affine horizon" of $\mathfrak{M}$ ) we distinguish two substructures corresponding to two possible sorts of lines. Let us set $\mathcal{L}_{k, m}^{\tau}:=\left\{[H, B]_{k}: H \in \mathcal{F}_{k-1, m}(\mathrm{Q}, W), B \in \mathcal{F}_{k+1, m+1}(\mathrm{Q}, W)\right\}$; it is seen that $\mathcal{L}_{k, m}^{\tau}=\left\{\bar{L}: L \in \mathcal{A}_{k, m}\right\}$. Note evident relation:

$\left\{L: L\right.$ is a line of $\left.\mathbf{P}_{k}(\mathrm{Q}), L \subset \mathcal{R}\right\}=\mathcal{L}_{k, m}^{\alpha} \cup \mathcal{L}_{k, m}^{\omega} \cup \mathcal{L}_{k, m+1}^{\alpha} \cup \mathcal{L}_{k, m+1}^{\omega} \cup \mathcal{L}_{k, m}^{\tau}$.

We define (write: $-\alpha=\omega,-\omega=\alpha$ )

$$
\mathfrak{N}^{\sigma}:=\left\langle\mathcal{R}, \mathcal{L}_{k, m}^{\sigma} \cup \mathcal{L}_{k, m}^{\tau} \cup \mathcal{L}_{k, m+1}^{-\sigma}\right\rangle \text { with } \sigma \in\{\alpha, \omega\} .
$$

Evidently, $\mathfrak{M}^{\sigma}$ can be embedded into $\mathfrak{N}^{\sigma}$. Intuitively, while the structure

$$
\left\langle\mathcal{R},\left\{L: L \text { is a line of } \mathbf{P}_{k}(\mathrm{Q}), L \subset \mathcal{R}\right\}\right\rangle
$$

can be considered as a projective completion of $\mathfrak{M}$ and, under specific assumptions, it is definable in $\mathfrak{M}, \mathfrak{N}^{\sigma}$ is a projective completion of $\mathfrak{M}^{\sigma}$.

To close this part it is worth to note the following analogue of Remark 5.3 and, at the same time, an analogue of [17, Fact 3.1].

Remark 6.10. Assume (2) and (4) from Theorem 6.9.

(i) If $m>0$ (cf. Remark 5.3) then the structure $\mathfrak{N}^{\omega}$ is definable in $\mathfrak{M}^{\omega}$.

(ii) If for each affine line $L=\mathbf{p}(H, B)$ there is a maximal isotropic $Y$ such that $B \subset Y$ and $\operatorname{dim}(W \cap Y) \geq r-k+m-3$ (cf. Remark 5.3) and $w<n+m-2 k$ (cf. Lemma 6.3), then the structure $\mathfrak{N}^{\alpha}$ is definable in $\mathfrak{M}^{\alpha}$.

According to Corollary 2.5 and Lemma 6.3, under condition $w<n+m-2 k$ each point of $\mathbf{A}_{k, m+1}(\mathrm{Q}, W)$ is a direction of a line in $\mathfrak{M}$ and each line of $\mathbf{A}_{k, m+1}(\mathrm{Q}, W)$ is a direction of a plane in $\mathfrak{M}$. This observation leads to the following.

Proposition 6.11. If $w<n+m-2 k$, then the horizon $\mathbf{A}_{k, m+1}(\mathrm{Q}, W)$ of $\mathfrak{M}$ can be defined in terms of $\mathfrak{A}$.

Finally, the question arises whether the adjacency of $\mathfrak{M}$ is definable purely in terms of the geometry of $\mathfrak{A}$ ? Unfortunately, the answer is not straightforward. The reasoning for spine spaces that justifies [17, Proposition 4.12], based on the fact that two distinct stars or tops of $\mathfrak{A}$ share no line on the horizon, cannot be adopted here without significant alterations. Note that if $\mathcal{L}^{\omega} \cup \mathcal{L}^{\alpha}=\emptyset$, then practically $\mathfrak{A}=\mathfrak{M}$. Therefore we assume that $\mathcal{L}^{\omega} \cup \mathcal{L}^{\alpha} \neq \emptyset$.

Theorem 6.12. If the ground field of $\mathbb{V}$ is of odd characteristic, then the structure $\mathfrak{M}$ can be defined in terms of $\mathfrak{A}$.

Proof. The proof is divided into several steps. For distinct points $U_{1}, U_{2}$ of $\mathfrak{M}$ we define

$$
\begin{aligned}
U_{1} \sim^{+} U_{2} & : \Longleftrightarrow U_{1}, U_{2} \subset B \text { for some } B \in \mathcal{F}_{k+1, m+1}(\mathrm{Q}, W), \\
U_{1} \sim_{-} U_{2} & : \Longleftrightarrow H \subset U_{1}, U_{2} \text { for some } H \in \mathcal{F}_{k-1, m}(\mathrm{Q}, W), \\
U_{1} \sim U_{2} & : \Longleftrightarrow U_{1} \sim^{+} U_{2} \text { or } U_{1} \sim_{-} U_{2} .
\end{aligned}
$$

Note that $U_{1} \sim^{+} U_{2}$ yields that either $U_{1} \lambda^{\text {at }} U_{2}$ or $U_{1} \lambda^{\omega} U_{2}$, while $U_{1} \sim_{-} U_{2}$ yields that either $U_{1} \lambda^{\text {af }} U_{2}, U_{1} \lambda^{\alpha} U_{2}$, or $U_{1}, U_{2}$ are not collinear in $\mathfrak{M}$. 
Step 1. The following conditions are equivalent.

(i) $U_{1} \sim^{+} U_{2}$ or $U_{1} \sim_{-} U_{2}$.

(ii) There is a plane $\Pi_{1}$ through $U_{1}$ parallel to a plane $\Pi_{2}$ through $U_{2}$ in $\mathfrak{A}$.

Proof of Step 1. (i) $\Longrightarrow$ (ii): Assume that $U_{1}, U_{2} \subset B \in \mathcal{F}_{k+1, m+1}(\mathrm{Q}, W)$, then $\mathrm{T}(B)$ is a semiaffine space (of the form $\mathcal{T}^{\omega}$ ) and one easily finds $\Pi_{1}, \Pi_{2}$ in it.

Next, assume that $U_{1}, U_{2} \supset H \in \mathcal{F}_{k-1, m}(\mathrm{Q}, W)$. Set $B:=U_{1}+U_{2}$. If $B \in \mathrm{Q}$ then $L=\mathbf{p}(H, B)$ is a line of $\mathfrak{M}$. Applying analogous reasoning we find $\Pi_{1}, \Pi_{2}$ in an extension $[H, Y]_{k}$ of the type $\mathcal{S}^{\alpha}$. If $B \notin \mathrm{Q}$ then, in any case $L$ is a line of the surrounding $\mathbf{A}_{k, m}(\mathbb{V}, W)$. Let us restrict to the subspaces around $H$; they form a spine space in the projective space $\mathbf{P}_{1}(\mathbb{V} / H)$ with the quadric $\mathrm{Q}(\xi / H)$ distinguished. Projective reasoning proves that required planes $\Pi_{1}, \Pi_{2}$ exist.

(ii) $\Longrightarrow$ (i): Let $\Pi_{i}$ be parallel planes of $\mathfrak{A}$ with $U_{i} \in \Pi_{i}, i=1,2$. Let $L_{0}=\Pi_{1}^{\infty}=\Pi_{2}^{\infty}$ be the improper line of $\Pi_{i}$. Then $L_{0} \in \mathcal{L}_{k, m+1}^{\alpha}$ or $L_{0} \in \mathcal{L}_{k, m+1}^{\omega}$. In the first case $L_{0}, U_{1}, U_{2}$ are contained in the (unique) extension to a top $\mathrm{T}(B)$ with $B \in \mathcal{F}_{k+1, m+1}(\mathrm{Q}, W)$ and therefore $U_{1} \sim^{+} U_{2}$. In the second case extensions of $\Pi_{i}$ to maximal strong subspaces have form $\left[H, Y_{i}\right]_{k}$ (they have $L_{0}$ in common), where $H \in \mathcal{F}_{k-1, m}(\mathrm{Q}, W)$. So, $U_{1} \sim_{-} U_{2}$. $\diamond$

Let us write

$$
\mathfrak{M}_{0}:=\mathbf{A}_{k, m}(\mathbb{V}, W) \uparrow \mathcal{F}_{k, m}(\mathrm{Q}, W)
$$

for the surrounding spine space with point set restricted to totally isotropic subspaces. Note that the distinction between $\mathfrak{M}$ and $\mathfrak{M}_{0}$ consists in the range of their line sets. More precisely, for a line $L=\mathbf{p}(H, B)$ of $\mathfrak{M}_{0}$ its base $B$ needs not to be totally isotropic and

$$
L \text { is a line of } \mathfrak{M} \quad \text { iff } \quad|L| \geq 3 .
$$

Step 2. Let $U_{1}, U_{2} \in \mathcal{F}_{k, m}(\mathrm{Q}, W)$ and $U_{1} \neq U_{2}$. The following conditions are equivalent.

(i) $U_{1} \sim U_{2}$.

(ii) $U_{1}, U_{2}$ are collinear in $\mathfrak{M}_{0}$ with exception when the line $L$ of $\mathfrak{M}_{0}$ which joins them has form $L=\mathbf{p}(H, B)$ where $H \in \mathcal{F}_{k-1, m-1}(\mathrm{Q}, W), B \in \mathcal{F}_{k+1, m+1}(\mathbb{V}, W)$, and $B \notin \mathrm{Q}$ (i.e. $L$ is an $\omega$-line in $\mathbf{A}_{k, m}(\mathbb{V}, W)$ ).

Proof of Step 2. (i) $\Longrightarrow$ (ii): It is clear that $U_{1}, U_{2}$ are collinear in the surrounding Grassmann space. If $U_{1} \sim{ }^{+} U_{2}$, then they lie on an affine or $\omega$-line in $\mathfrak{M}$ by Table 1 , while if $U_{1} \sim_{-} U_{2}$, then they lie on an affine or $\alpha$-line in $\mathfrak{M}_{0}$.

(ii) $\Longrightarrow$ (i): Now, let $U_{1}, U_{2}$ be collinear in $\mathfrak{M}_{0}$. Hence $U_{1}, U_{2} \in \mathbf{p}(H, B)$ for suitable $H, B$. If $\operatorname{dim}(B \cap W)=m$, then $\operatorname{dim}(H \cap W)=m$ and thus $U_{1} \sim_{-} U_{2}$. If $\operatorname{dim}(B \cap W)=$ $m+1$, then two cases arise: $\operatorname{dim}(H \cap W)=m, m-1$. In the former we have $U_{1} \sim_{-} U_{2}$. In the later $H \in \mathcal{F}_{k-1, m-1}(\mathrm{Q}, W)$ and $B \in \mathcal{F}_{k+1, m+1}(\mathbb{V}, W)$. If $B \in \mathrm{Q}$, then $U_{1} \sim^{+} U_{2}$, otherwise we get the excluded case.

Step 3. A set $X$ of points of $\mathfrak{A}$ is a maximal at least 3 -element $\sim$-clique iff $X$ has one of the following forms:

(a) $X=\mathrm{T}(B)$ for some $B \in \mathcal{F}_{k+1, m+1}(\mathrm{Q}, W)$,

(b) $X=\mathrm{T}(B)$ for some $B \in \mathcal{F}_{k+1, m}(\mathrm{Q}, W)$, 
(c) $X=\mathrm{S}(H)$ for some $H \in \mathcal{F}_{k-1, m}(\mathrm{Q}, W)$, or

(d) $X=[H, Y] \cap \mathcal{F}_{k, m}(\mathrm{Q}, W)$ for some $H \in \mathcal{F}_{k-1, m-1}(\mathrm{Q}, W)$ and $H \subset Y \in \mathrm{Q}_{r}$.

Proof of Step 3. It is easy to verify that sets defined in (a)-(d) are maximal $\sim$-cliques. Now, let $X$ be a maximal at least 3-element $\sim$-clique. In view of Step 2, $X$ is a subset of a clique in $\mathfrak{M}_{0}$. So, we need general tops $\mathrm{T}_{0}(B)=[\Theta, B]_{k}$ for $B \in \operatorname{Sub}_{k+1}(\mathbb{V})$ and stars $\mathrm{S}_{0}(H)=[H, V]_{k}$ for $H \in \operatorname{Sub}_{k-1}(\mathbb{V})$. Let us examine the following four cases:

$X \subseteq \mathrm{T}_{0}(B), B \in \mathcal{F}_{k+1, m+1}(\mathbb{V}, W)$

If $B \in \mathrm{Q}$, then any two points of $\mathfrak{M}_{0}$ in $\mathrm{T}(B)$ are $\sim^{+}$-adjacent and thus $X=$ $\mathrm{T}(B) \cap \mathcal{F}_{k, m}(\mathrm{Q}, W)$ is a $\sim$-clique as in (a). If $B \notin \mathrm{Q}$, then $|X| \leq 2$ by [19, Proposition 4.4], a contradiction.

$X \subseteq \mathrm{T}_{0}(B), B \in \mathcal{F}_{k+1, m}(\mathbb{V}, W)$

Since $|X| \geq 3$ we have $B \in \mathrm{Q}$ by [19, Proposition 4.4]. Any two points of $\mathfrak{M}_{0}$ in $\mathrm{T}(B)$ are $\sim_{-}$-adjacent, so $X=\mathrm{T}(B) \cap \mathcal{F}_{k, m}(\mathrm{Q}, W)$ has form (b).

$X \subseteq \mathrm{S}_{0}(H), H \in \mathcal{F}_{k-1, m}(\mathbb{V}, W)$

Note that $H \in \mathrm{Q}$ as $X$ is nonempty. This implies that any two points of $\mathfrak{M}_{0}$ in $\mathrm{S}(H)$ are $\sim_{-}$-adjacent. Consequently, $X=\mathrm{S}(H) \cap \mathcal{F}_{k, m}(\mathrm{Q}, W)$ has form (c).

$X \subseteq \mathrm{S}_{0}(H), H \in \mathcal{F}_{k-1, m-1}(\mathbb{V}, W)$

As above $H \in \mathrm{Q}$. The points of $\mathfrak{M}_{0}$ in $\mathrm{S}_{0}(H)$ are $\sim$-adjacent iff they are $\sim^{+}$adjacent i.e. they are collinear in the surrounding polar Grassmann space where the appropriate clique has form $[H, Y]_{k}$ for some $Y \in \mathrm{Q}_{r}$ (cf. [7, Section 3]). Hence $X=[H, Y]_{k} \cap \mathcal{F}_{k, m}(\mathrm{Q}, W)$ has form (d).

That way we obtain the desired list (a) - (d).

Note that the $\lambda^{\text {af }}$-cliques are essentially smaller than $\sim$-cliques.

Step 4. At least 3 -element minimal intersections of the maximal $\sim$-cliques are lines of $\mathfrak{M}$.

Proof of Step 4. Let $\mathcal{K}_{\mathrm{x}}$ be the family of cliques of the form (x) defined in Step 3. Let $X_{1}, X_{2}$ be two distinct $\sim$-cliques and $Z=X_{1} \cap X_{2}$. If $X_{1}, X_{2} \in \mathcal{K}_{(\mathrm{a})} \cup \mathcal{K}_{(\mathrm{b})}, X_{1}, X_{2} \in$ $\mathcal{K}_{(\mathrm{c})}$, or $X_{1} \in \mathcal{K}_{(\mathrm{b})} \cup \mathcal{K}_{(\mathrm{c})}$ and $X_{2} \in \mathcal{K}_{(\mathrm{d})}$, then $Z$ contains at most a single point. If $X_{1} \in \mathcal{K}_{(\mathrm{a})}$ and $X_{2} \in \mathcal{K}_{(\mathrm{c})}$, then $Z$ is an affine line of $\mathfrak{M}$. If $X_{1} \in \mathcal{K}_{(\mathrm{b})}$ and $X_{2} \in \mathcal{K}_{(\mathrm{c})}$, then $Z$ is an $\alpha$-line of $\mathfrak{M}$. If either $X_{1} \in \mathcal{K}_{(\text {a) }}$ and $X_{2} \in \mathcal{K}_{(\mathrm{d})}$ or $X_{1}, X_{2} \in \mathcal{K}_{(\mathrm{d})}$, then at least 3-element minimal $Z$ is an $\omega$-line of $\mathfrak{M}$.

It is evident that every projective line of $\mathfrak{M}$ can be presented as the intersection of cliques enumerated in Step 3. So, applying Step 4 we get the line set of $\mathfrak{M}$ recovered which makes the proof of Theorem 6.12 complete.

Remark 6.13. The horizon of a star in $\mathfrak{M}$ may have strange properties. Assume that $W \in \mathrm{Q}$ and let $H \in \mathrm{Q}_{k-1}, H \subset W^{\perp}$. Set $m:=\operatorname{dim}(H \cap W)$. This means that $k-1+w-m<r$. Then there is an $Y_{0} \in \mathrm{Q}_{r}$ such that $H \cup W \subset Y_{0}$. So, $Y_{0} \cap W=W$. Write $S_{0}=\left[H, Y_{0}\right]_{k} \cap \mathcal{F}_{k, m}(\mathrm{Q}, W)$. Then $S_{0}^{\infty}=[H, H+W]_{k}$. Take any $S=[H, Y]_{k} \cap$ $\mathcal{F}_{k, m}(\mathrm{Q}, W)$ contained in $\mathrm{S}(H)$. Then $S^{\infty}=[H, H+(W \cap Y)]_{k} \subset[H, H+W]_{k}=S_{0}^{\infty}$. So, in this case

$\mathrm{S}(H)^{\infty}$ is the projective space $[H, H+W]_{k}$ contained in $\mathfrak{M}^{\infty}$. 
Nevertheless, $\mathrm{S}(H)$ contains affine subspaces of different dimensions.

Note that in this case $k-m-1=\operatorname{dim}\left(\mathrm{T}(B)^{\infty}\right)=\operatorname{dim}\left(\mathrm{S}(H)^{\infty}\right)=w-m-1$ yields $w=k$, so horizons of stars and tops may have equal dimensions only when $\mathfrak{M}$ consists of points in $\mathrm{Q}_{k}$ that are at the fixed distance $k-m$ from the fixed point $W$.

Remark 6.14. Theorem 6.12 for polar spine spaces and its counterpart [17, Proposition 4.12] for spine spaces both say that the respective geometry depends only on affine lines together with parallelism, that is, projective lines can be recovered using affine line structure. However, the idea of the proof presented in this paper is more general than that in [17] because it does not rely on specific horizons and intersections of stars which are completely different in $\mathfrak{M}$ and in $\mathbf{A}_{k, m}(\mathbb{V}, W)$. As such it can be applied for spine spaces and is expected to give less complex reasonings.

\section{Classifications}

Table 1: The classification of lines in a polar spine space $\mathbf{A}_{k, m}(\mathrm{Q}, W)$.

\begin{tabular}{ccc}
\hline Class & Representative line $g=\mathbf{p}(H, B) \cap \mathcal{F}_{k, m}(\mathrm{Q}, W)$ & $g^{\infty}$ \\
\hline $\mathcal{A}_{k, m}(\mathrm{Q}, W)$ & $H \in \mathcal{F}_{k-1, m}(\mathrm{Q}, W), B \in \mathcal{F}_{k+1, m+1}(\mathrm{Q}, W)$ & $H+(B \cap W)$ \\
$\mathcal{L}_{k, m}^{\alpha}(\mathrm{Q}, W)$ & $H \in \mathcal{F}_{k-1, m}(\mathrm{Q}, W), B \in \mathcal{F}_{k+1, m}(\mathrm{Q}, W)$ & - \\
$\mathcal{L}_{k, m}^{\omega}(\mathrm{Q}, W)$ & $H \in \mathcal{F}_{k-1, m-1}(\mathrm{Q}, W), B \in \mathcal{F}_{k+1, m+1}(\mathrm{Q}, W)$ & - \\
\hline
\end{tabular}

Each strong subspace $X$ of a polar spine space is a slit space, that is a projective space $\mathbf{P}$ with a subspace $\mathcal{D}$ removed. In the extremes $\mathcal{D}$ can be void, then $X$ is basically a projective space, or a hyperplane, then $X$ is an affine space.

Table 2: The classification of stars and tops in a polar spine space $\mathbf{A}_{k, m}(\mathrm{Q}, W)$.

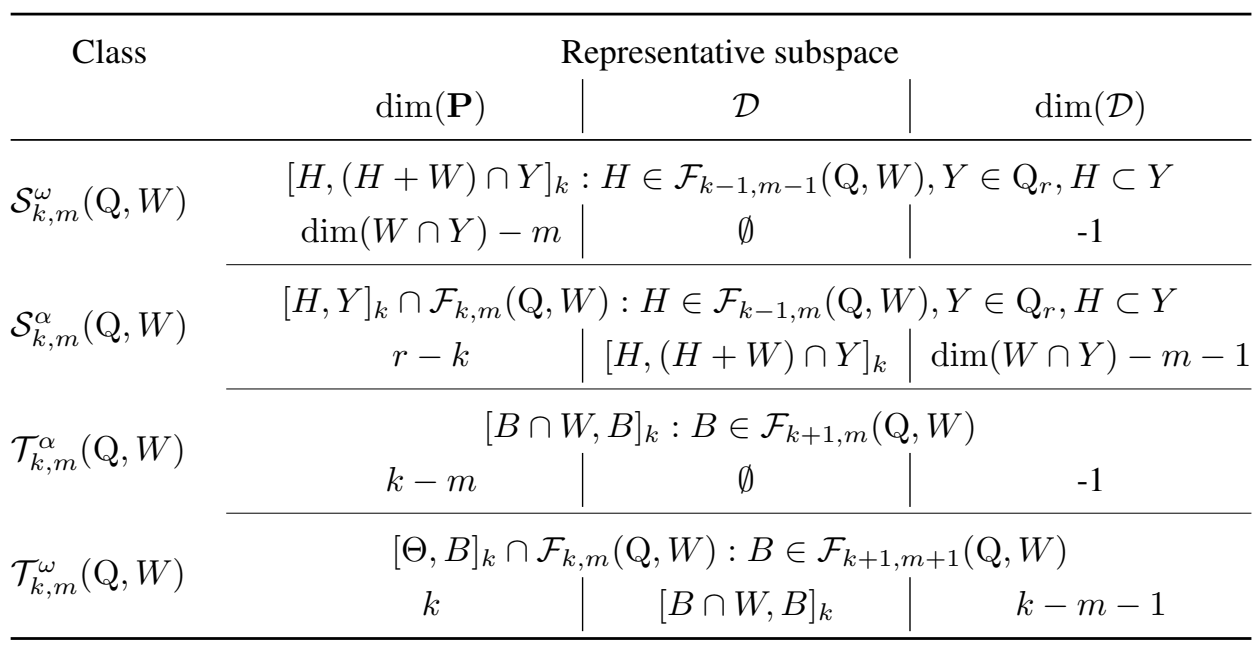




\section{ORCID iDs}

Krzysztof Petelczyc (D) https://orcid.org/0000-0003-0500-9699

Krzysztof Prażmowski iD https://orcid.org/0000-0002-5352-5973

Mariusz Żynel iD https://orcid.org/0000-0001-9297-4774

\section{References}

[1] A. Ben-Tal and A. Ben-Israel, Ordered incidence geometry and the geometric foundations of convexity theory, J. Geom. 30 (1987), 103-122, doi:10.1007/bf01227810.

[2] A. M. Cohen, Point-line spaces related to buildings, in: Handbook of Incidence Geometry, North-Holland, Amsterdam, pp. 647-737, 1995, doi:10.1016/b978-044488355-1/50014-1.

[3] A. M. Cohen and E. E. Shult, Affine polar spaces, Geom. Dedicata 35 (1990), 43-76, doi: 10.1007/bf00147339.

[4] H. Karzel and I. Pieper, Bericht über geschlitzte inzidenzgruppen, Jber. Deutsch. Math.-Verein 72 (1970), 70-114, https: / / eudml.org/doc/146588.

[5] M. Pankov, Grassmannians of Classical Buildings, volume 2 of Algebra and Discrete Mathematics, World Scientific Publishing, Hackensack, NJ, 2010, doi:10.1142/7844.

[6] M. Pankov, K. Prażmowski and M. Żynel, Transformations preserving adjacency and base subsets of spine spaces, Abh. Math. Sem. Univ. Hamburg 75 (2005), 21-50, doi:10.1007/ bf02942034.

[7] M. Pankov, K. Prażmowski and M. Żynel, Geometry of polar Grassmann spaces, Demonstratio Math. 39 (2006), 625-637, doi:10.1515/dema-2006-0318.

[8] K. Petelczyc, K. Prażmowski and M. Żynel, Geometry on the lines of polar spine spaces, Aequationes Math. 94 (2020), 829-846, doi:10.1007/s00010-020-00733-2.

[9] K. Petelczyc and M. Żynel, Geometry on the lines of spine spaces, Aequationes Math. 92 (2018), 385-400, doi:10.1007/s00010-017-0523-6.

[10] K. Petelczyc and M. Żynel, The complement of a subspace in a classical polar space, Ars Math. Contemp. 17 (2019), 447-454, doi:10.26493/1855-3974.1917.ea5.

[11] M. Prażmowska, K. Prażmowski and M. Żynel, Affine polar spaces, their Grassmannians, and adjacencies, Math. Pannon. 20 (2009), 37-59, http: / / mathematica-pannonica . ttk.pte.hu/articles/mp20-1/mpprazy.pdf.

[12] K. Prażmowski, On a construction of affine Grassmannians and spine spaces, J. Geom. 72 (2001), 172-187, doi:10.1007/s00022-001-8579-8.

[13] K. Prażmowski and M. Żynel, Automorphisms of spine spaces, Abh. Math. Sem. Univ. Hamburg 72 (2002), 59-77, doi:10.1007/bf02941665.

[14] K. Prażmowski and M. Żynel, Affine geometry of spine spaces, Demonstratio Math. 36 (2003), 957-969, doi:10.1515/dema-2003-0420.

[15] K. Prażmowski and M. Żynel, Geometry of the structure of linear complements, J. Geom. 79 (2004), 177-189, doi:10.1007/s00022-003-1446-z.

[16] K. Prażmowski and M. Żynel, Extended parallelity in spine spaces and its geometry, J. Geom. 85 (2006), 110-137, doi:10.1007/s00022-005-0032-y.

[17] K. Prażmowski and M. Żynel, Possible primitive notions for geometry of spine spaces, J. Appl. Log. 8 (2010), 262-276, doi:10.1016/j.jal.2010.05.001.

[18] K. Radziszewski, Subspaces and parallelity in semiaffine partial linear spaces, Abh. Math. Sem. Univ. Hamburg 73 (2003), 131-144, doi:10.1007/bf02941272.

[19] M. Żynel, Finite Grassmannian geometries, Demonstratio Math. 34 (2001), 145-160, doi:10. 1515/dema-2001-0118. 